\title{
RESEARCH
}

Open Access

\section{High-intensity exercise and cognitive function in cognitively normal older adults: a pilot randomised clinical trial}

Belinda M. Brown ${ }^{1,2,3,4^{*}}$ DD, Natalie Frost ${ }^{5}$, Stephanie R. Rainey-Smith ${ }^{2,3,4,5}$, James Doecke ${ }^{6,7}$, Shaun Markovic ${ }^{1,2}$, Nicole Gordon ${ }^{1}$, Michael Weinborn ${ }^{2,5}$, Hamid R. Sohrabi ${ }^{2,3,4,7}$, Simon M. Laws ${ }^{4,8,9}$, Ralph N. Martins ${ }^{2,4,7}$, Kirk I. Erickson ${ }^{1,10}$ and Jeremiah J. Peiffer ${ }^{1,3}$

\begin{abstract}
Background: Physical inactivity has been consistently linked to increased risk of cognitive decline; however, studies examining the impact of exercise interventions on cognition have produced inconsistent findings. Some observational studies suggest exercise intensity may be important for inducing cognitive improvements; however, this has yet to be thoroughly examined in older adult cohorts. The objective of the current study was to evaluate the effect of systematically manipulated high-intensity and moderate-intensity exercise interventions on cognition.
\end{abstract}

Methods: This multi-arm pilot randomised clinical trial investigated the effects of 6 months of high-intensity exercise and moderate-intensity exercise, compared with an inactive control, on cognition. Outcome measures were assessed at pre(baseline), post- (6 months), and 12 months post-intervention. Ninety-nine cognitively normal men and women (aged 60-80 years) were enrolled from October 2016 to November 2017. Participants that were allocated to an exercise group (i.e. highintensity or moderate-intensity) engaged in cycle-based exercise two times per week for 6 months. Cognition was assessed using a comprehensive neuropsychological test battery. Cardiorespiratory fitness was evaluated by a graded exercise test.

Results: There was a dose-dependent effect of exercise intensity on cardiorespiratory fitness, whereby the high-intensity group experienced greater increases in fitness than the moderate-intensity and control groups. However, there was no direct effect of exercise on cognition.

Conclusions: We did not observe a direct effect of exercise on cognition. Future work in this field should be appropriately designed and powered to examine factors that may contribute to individual variability in response to intervention.

Trial registration: This study is registered with the Australian New Zealand Clinical Trials Registry (ACTRN12617000643370). Registered on 3 May 2017—retrospectively registered. https:/www.anzctr.org.au/Trial/Registration/TrialReview.aspx?id=372 780

Keywords: Exercise, Cognition, Intensity, Genetics, Dementia, Cardiorespiratory fitness

\footnotetext{
* Correspondence: b.brown@murdoch.edu.au

'Discipline of Exercise Science, College of Science, Health, Engineering and Education, Murdoch University, 90 South Street, Murdoch, WA 6150, Australia

${ }^{2}$ Australian Alzheimer's Research Foundation, Sarich Neuroscience Research Institute, Nedlands, Western Australia, Australia

Full list of author information is available at the end of the article
}

(c) The Author(s). 2021 Open Access This article is licensed under a Creative Commons Attribution 4.0 International License, which permits use, sharing, adaptation, distribution and reproduction in any medium or format, as long as you give appropriate credit to the original author(s) and the source, provide a link to the Creative Commons licence, and indicate if changes were made. The images or other third party material in this article are included in the article's Creative Commons licence, unless indicated otherwise in a credit line to the material. If material is not included in the article's Creative Commons licence and your intended use is not permitted by statutory regulation or exceeds the permitted use, you will need to obtain permission directly from the copyright holder. To view a copy of this licence, visit http://creativecommons.org/licenses/by/4.0/ The Creative Commons Public Domain Dedication waiver (http://creativecommons.org/publicdomain/zero/1.0/) applies to the data made available in this article, unless otherwise stated in a credit line to the data. 


\section{Introduction}

Physical inactivity is considered one of the greatest modifiable risk factors for dementia [1]; however, attempts to provide definitive evidence from randomised controlled trials (RCTs) of a link between exercise and enhanced cognition have been inconsistent. Indeed, a 2015 Cochrane review [2] of RCTs concluded there is insufficient evidence, in cognitively normal older adults, to suggest an effect of exercise on cognition. While a more recent meta-analysis [3], assessing a similar cohort, identified positive effects of exercise when session durations were in excess of $45 \mathrm{~min}$ and at least of moderate intensity. These conflicting results indicate the need for greater clarity for the use of exercise as a method for preventing cognitive decline, specifically the precise parameters needed for improving brain health.

When compared with the total volume of physical activity, observational work has reported a stronger association between objectively measured intensity of physical activity and cognitive function [4, 5]. Furthermore, acute bouts of high-intensity exercise improve memory and executive function to a greater extent than moderateintensity continuous exercise bouts [6, 7]. Although previous work in the area is promising, the importance of exericise intensity in enhancing cognitive health requires rigorous examination in RCTs. A recent 12-week intervention in older adults demonstrated greater improvements in memory after undertaking high-intensity compared with moderate-intensity exercise, or a stretching control [8]. The use of high-intensity exercise is safe in older populations [9] and provides a time-effective method to increase physical health, yet, until more consistent and rigorous evidence is available, the widespread use of high-intensity exercise to enhance cognitive health will continue to be questioned.

Variability across studies might also be explained by factors moderating exercise-induced changes in cognition. Genetic factors, such as the apolipoprotein $\mathrm{E}$ $(A P O E) \varepsilon 4$ allele and the brain-derived neurotrophic factor $(B D N F)$ Val66Met single nucleotide polymorphism, may modulate the relationship between exercise and brain health [10-12]. The literature in these fields is predominantly sourced from observational studies, which have contributed to largely inconsistent findings. In addition, variability in cognitive response may be due to variability in cardiorespiratory fitness change following exercise [13, 14]. Evidence from RCTs is needed to gain a greater understanding of these potential mediating and moderating effects on cognition.

The current pilot RCT was designed to provide a head-to-head comparison of work-matched moderateintensity and high-intensity exercise on cognition in cognitively normal older adults. We hypothesised that both intervention groups would receive benefits to cognition, but the high-intensity group would receive additional benefit beyond the moderate-intensity group in a dosedependent fashion. Based on the hypothesis that increases in cardiorespiratory fitness are an important factor in the relationship between exercise and cognition, we investigated whether change in fitness is associated with improved cognition. Finally, we conducted an exploratory investigation as to whether targeted genetic factors ( $A P O E$ \&4 carriage and BDNF Val66Met) moderate the effect of exercise on cognition, and the relationship between altered fitness and cognitive changes. Based on previous literature, we hypothesised that $A P O E$ $\varepsilon 4$ carriers and $B D N F$ Val66Met carriers would receive the greatest benefit from exercise, in terms of cognitive improvements $[10,12]$.

\section{Methods \\ Trial design}

The Intense Physical Activity and Cognition (IPAC) study was a single-site parallel pilot randomised controlled trial conducted between October 2016 and September 2019 at Murdoch University and the Australian Alzheimer's Research Foundation, Western Australia. An open access protocol paper for the IPAC study has been published previously [15]. Participants were randomised to either 6 months of supervised high-intensity exercise, supervised moderate-intensity exercise, or an inactive control group. Reporting of this study adheres to Consolidated Standards of Reporting Trials (CONSORT) guidelines [16].

The IPAC study is registered with the Australian New Zealand Clinical Trials Registry (ACTRN12617000643370). The human research ethics committees at Murdoch University and Edith Cowan University approved the conduct of this study, and all participants provided written informed consent.

\section{Participants and randomisation}

Participants were recruited between October 2016 and November 2017 from a number of sources, including media advertisement, flyers, and word-of-mouth. A full list of inclusion and exclusion criteria have been described previously [15]. Our power analysis was based on the primary outcome of cognitive function: using data from Vidoni et al. [13], we required 28 participants per group to detect differences between three groups at $80 \%$ power and the $5 \%$ level of significance. A block randomisation protocol (conducted by a researcher who was not collecting outcome data) was used to randomly assign participants to one of the following three groups: high-intensity exercise, moderate-intensity exercise, or a control group. 


\section{Interventions}

Participants that were allocated to an exercise group (i.e. high-intensity or moderate-intensity) engaged in exercise two times per week (under the supervision of an Accredited Exercise Physiologist) for 6 months. Each exercise session lasted $50 \mathrm{~min}$ and was conducted on a cycle ergometer (Wattbike Pro; Wattbike, Australia). Target intensity was set using the 6 to 20 Borg Scale of Perceived Exertion [17]. Further details of the exercise interventions can be found within the supplementary material.

Adherence to the intervention was measured via session attendance. In addition, exercise intensity was calculated for each participant in the moderate- and highintensity groups: the percentage of peak aerobic power (measured via a graded exercise test) was calculated for each session (not including the warm-up, cool-down, and for the high-intensity group, the recovery between intervals). The percentage of peak aerobic power in the initial 3 months were calculated using baseline peak aerobic power output, while months 4 to 6 were calculated using peak aerobic power output from the midintervention fitness test.

Participants randomised to the control group were provided with an information session regarding the benefits of exercise for overall physical health and known benefits to the brain. Participants in the control group did not receive any exercise instructions.

\section{Procedures and outcome measures}

Full methodological detail regarding outcome measures can be found in the supplementary material.

\section{Cognitive assessment}

A comprehensive battery of neuropsychological tests was administered to all participants at baseline, 6 months, and 18 months. Composite scores for global cognitive function, attention, episodic memory, and executive function were calculated.

The battery included the Montreal Cognitive Assessment (MoCA), Wechsler Adult Intelligence Scale-III Digit Span, California Verbal Learning Test (CVLT-II), Brief Visual Memory Test (BVMT), Trail Making Test forms A and B, and the NIH EXAMINER Verbal fluency task, Flanker, and Set-shifting. A computerised Cogstate battery (www.cogstate.com) was administered including Groton Maze learning and recall, and identification, detection, one-card learning, and one-back tasks.

We calculated cognitive composite scores using $z$ scores of individual's performances across all timepoints. For scores where a lower score indicates better performance (i.e. speed), we inversed the score ([score $\left.]^{*}-1\right)$. The composite scores included the following tasks: (1) Global cognitive composite: Digit Span, Cogstate one-back, Cogstate identification task, CVLT (learning, short delay recall, long delay recall, and recognition d'), BVMT (learning and long delay recall), Cogstate Groton Maze recall, Trails B, Phonemic fluency, Flanker, and Setshifting; (2) Attention: Digit Span (Forward only) and Cogstate identification task; (3) Episodic Memory: CVLT (short delay recall, long delay recall, and recognition d'), BVMT long delay recall, and Groton Maze recall; and (4) Executive function: Trails B, Phonemic fluency, Flanker, and Set-shifting.

\section{Physical assessment}

At baseline, 3, 6, and 18 months, all participants underwent a cycling-based graded exercise test to quantify peak aerobic capacity $\left(\mathrm{VO}_{2}\right.$ peak) and peak power. All participants also underwent a dual-energy X-ray absorptiometry (DXA) scan, using a Hologic Discovery Bone Densitometer (Hologic, USA), in order to quantify volume of fat, muscle, and bone tissue in the body.

\section{Genotyping}

TaqMan genotyping assays were performed [18] to determine APOE genotype (rs7412, assay ID: C__904973_10; rs429358, assay ID: C__3084793_20) and BDNF Val66Met single nucleotide polymorphism (rs6265, assay ID: C_11592758_10). Dichotomous variables indicating $A P O E \& 4$ carriers or non-carriers and BDNF Val66Met carriers or non-carriers were created.

\section{Statistical methods}

Analyses were conducted in $\mathrm{R}$ statistical computing packages version 3.6.2 [19] and Statistical Package for the Social Sciences Version 24 (IBM). Data were inspected to determine parametric testing was appropriate for all physiological and cognitive variables.

\section{Descriptive statistics}

Descriptive statistics were calculated to compare baseline information across study groups. Analyses of variance (for continuous variables) and chi-square analyses (for categorical variables) were conducted to identify differences.

\section{Intervention group analysis}

All participants that completed a baseline assessment were included in the intention-to-treat (ITT) analyses, regardless of adherence to session attendance, or study withdrawal. To examine the effect of study group on cognition over time, a series of linear mixed models (LMMs) were conducted. Repeated cognitive composite scores were entered as dependent variables, and age, gender, education, time (years), group, and time*group were entered as fixed factors, and participant identification number as a random factor, into the model. Post hoc group comparisons were conducted for any 
significant time"group interactions (high-intensity as reference group). LMMs were conducted for baseline and 6-month data only, and then again for baseline, 6-, and 18 -month data. We report raw mean change scores from baseline and 95\% confidence intervals, and unstandardized beta coefficients (B) and their standard error. A positive B represents a positive slope for the moderate/ high-intensity groups, compared with the control group.

We ran LMMs to investigate the moderating effects of $B D N F$ Val66Met and APOE \&4 carriage on cognitive performance over the intervention. Each LMM had an additional interaction of either $B D N F^{*}$ time" group or $A P O E$ "time" group entered into separate models.

\section{Individual variability analysis}

The relationships between change in cognition and change in cardiorespiratory fitness $\left(\mathrm{VO}_{2}\right.$ peak) from preto post-intervention within the high-intensity group only, and for all study participants, were examined.
Residualised change scores were generated by entering post-intervention score as the dependent variable and pre-intervention score as the independent variable. Linear models were run with the residualised cognitive change as the dependent variable and residualised fitness change as the independent variable (age, gender, and education as covariates) [20]. For all study participants, the linear models were re-run with the inclusion of either $B D N F^{*}$ fitness change or $A P O E^{*}$ fitness change. The cohort was then stratified by $B D N F$ Val66Met carriage or $A P O E \& 4$ carriage and the linear models re-run.

\section{Results}

One hundred and eight participants were enrolled, with ninety-nine completing all baseline assessments and subsequent randomisation to a study group (Fig. 1; descriptive data, Table 1). Seven participants withdrew during the 6-month intervention.

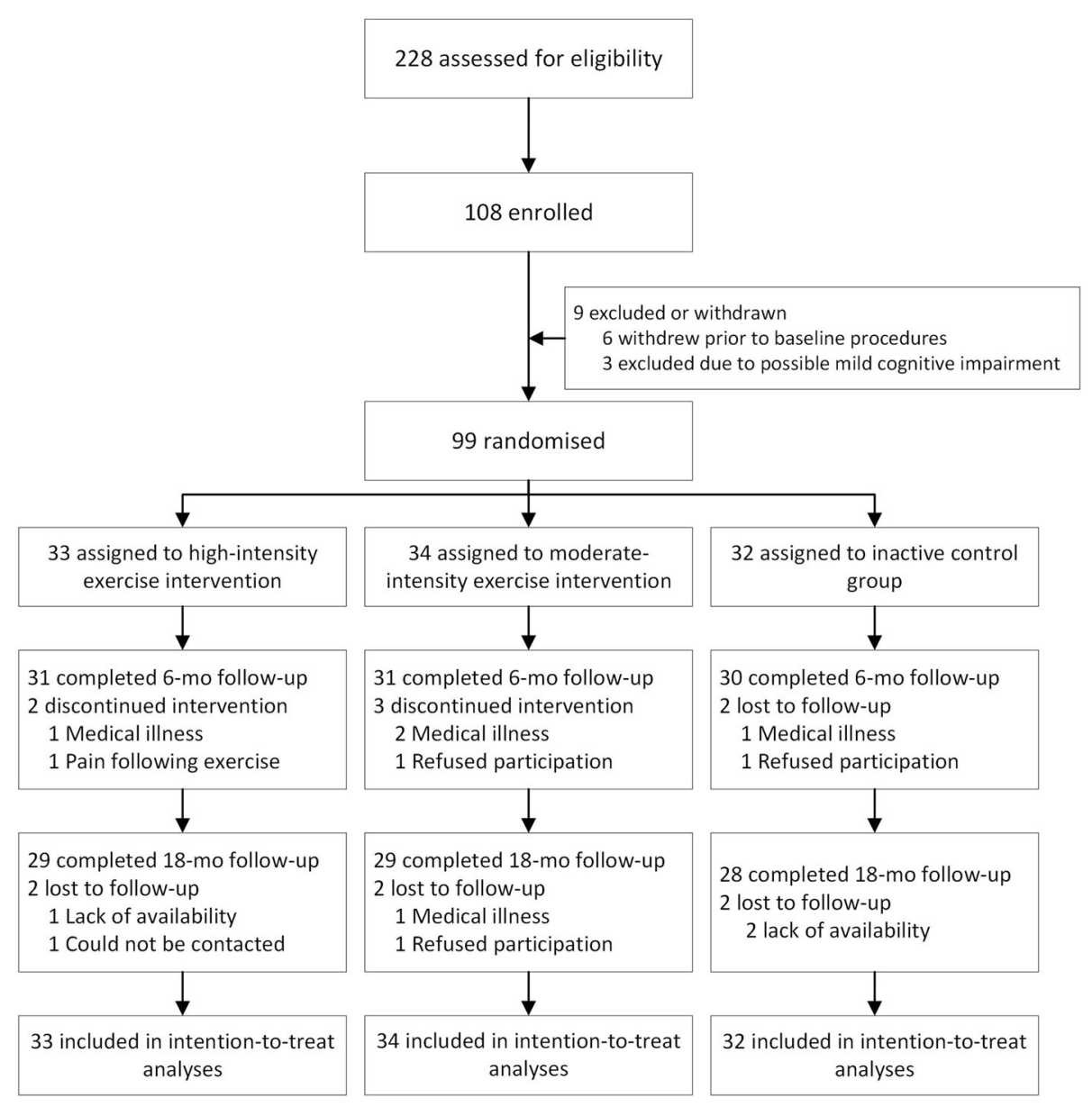

The number of cases may vary according to outcome measure due to missing data

Fig. 1 CONSORT diagram for Intense Physical Activity and Cognition study 
Table 1 Descriptive baseline characteristics of study cohort

\begin{tabular}{|c|c|c|c|c|}
\hline & High-intensity $(n=33)$ & Moderate-intensity $(n=34)$ & Control $(n=32)$ & Test statistic \\
\hline Age, years & $70.2 \pm 5.3$ & $68.4 \pm 4.2$ & $68.7 \pm 5.9$ & $F=1.22$ \\
\hline Gender, \% Female ( $n)$ & $51.5(17)$ & $52.9(18)$ & $59.4(19)$ & $x^{2}=0.79$ \\
\hline APOE $\varepsilon 4$ allele carriers, $\%(n)$ & $27.3(9)$ & $23.5(8)$ & $28.1(9)$ & $x^{2}=0.90$ \\
\hline BDNF Val66Met carriers, $\%(n)$ & $33.3(11)$ & $32.4(11)$ & $50(16)$ & $x^{2}=2.70$ \\
\hline Years of education & $13.5 \pm 2.2$ & $14.2 \pm 2.5$ & $14.5 \pm 2.1$ & $F=1.65$ \\
\hline Global cognition, MoCA score & $26.0 \pm 2.1$ & $26.4 \pm 2.8$ & $26.7 \pm 2.0$ & $F=0.64$ \\
\hline Baseline $\mathbf{V O}_{2}$ peak $(\mathrm{ml} / \mathrm{kg} / \mathrm{min})$ & $22.2 \pm 6.3$ & $24.7 \pm 6.9$ & $22.8 \pm 6.1$ & $F=1.36$ \\
\hline Baseline peak power $(\mathrm{W})$ & $128.9 \pm 49.4$ & $145.0 \pm 51.1$ & $126.4 \pm 37.1$ & $F=1.57$ \\
\hline Alcohol, Units per week & $5.7 \pm 5.9$ & $5.1 \pm 5.5$ & $6.4 \pm 6.1$ & $F=0.44$ \\
\hline Time from baseline to 6-mo assessment (wks) & $33.0 \pm 3.7$ & $34.7 \pm 4.7$ & $34.1 \pm 2.3$ & $F=1.60$ \\
\hline Time from baseline to 18 -mo assessment (wks) & $85.3 \pm 3.6$ & $88.3 \pm 5.6$ & $87.1 \pm 3.9$ & $F=3.38^{*}$ \\
\hline Physical activity (Met.min $/ \mathrm{wk}^{-1}$ ) & $4379 \pm 3708$ & $4372 \pm 2488$ & $3533 \pm 1981$ & $F=0.94$ \\
\hline DASS Depression score & $2.3 \pm 3.0$ & $1.61 \pm 2.1$ & $1.7 \pm 1.9$ & $F=0.95$ \\
\hline Daily kilojoule intake $^{a}$ & $6709 \pm 2459$ & $7430 \pm 3286$ & $6059 \pm 1896$ & $F=2.01$ \\
\hline BMI $\left(\mathrm{kg} / \mathrm{m}^{2}\right)$ & $25.8 \pm 3.7$ & $26.0 \pm 3.9$ & $25.3 \pm 3.4$ & $F=0.30$ \\
\hline Waist-hip ratio & $0.87 \pm 0.08$ & $0.88 \pm 0.07$ & $0.88 \pm 0.08$ & $F=0.08$ \\
\hline
\end{tabular}

${ }^{*} P<0.05$, post hoc Tukey's significant difference between high-intensity group and moderate-intensity group. Test statistics determined by one-way analysis of variance for continuous variables and chi-square for categorical variables. Abbreviations: $A P O E$ apolipoprotein $\mathrm{E}, B D N F$ Val66Met brain-derived neurotrophic factor Valine66Methionine single nucleotide polymorphism, $B M I$ body mass index, DASS Depression, Anxiety and Stress Scales, Met.min/wk ${ }^{-1}$ metabolic minutes per week (subjective habitual physical activity measurement), MoCA Montreal Cognitive Assessment, $\mathrm{VO}_{2}$ peak peak aerobic capacity (fitness measurement), $W$ wattage.

${ }^{a}$ Daily kilojoule intake quantified from the Cancer Council of Victoria Food Frequency Questionnaire

\section{Adherence to prescribed intervention}

There was no difference in exercise session attendance between the high-intensity $(85.5 \pm 12.4 \%)$ and moderateintensity $(86.3 \pm 9.8 \%)$ groups.

The high-intensity group maintained $120.6 \pm 25.1 \%$ of peak aerobic power during the high-intensity intervals, while the moderate-intensity group cycled continuously at $70.1 \pm 16.3 \%$ of peak aerobic power.

There were no serious adverse events recorded.

\section{Group comparisons}

A time"group effect was observed for cardiorespiratory fitness, peak aerobic power, and body fat from pre- to postintervention (Table 2). The high-intensity group experienced greater improvements in cardiorespiratory fitness $(+24.3 \%)$ compared with the moderate-intensity group $(+12.4 \% ; B=$ $3.92, p<0.01)$, and control group $(+2.4 \% ; B=7.36, p<$ $0.001)$. The high-intensity group also experienced greater improvements in peak aerobic power $(+29 \% ; B=55.62, p<$ $0.001)$ and decreases in percentage body fat $(-3.5 \% ; B=-$ $1.59, p<0.05$ ), compared with the control group (peak power change, $+1.3 \%$; percentage body fat change, $0.0 \%$ ).

There were no significant time"group effects on any of the cognitive composite scores. Main effects for time were significant for the executive function composite variable $(p<0.05)$, likely indicating a small practice effect experienced on the tasks assessing this cognitive domain.
Similarly, there were no significant effects of the genoty$p e^{*}$ time"group interactions on the cognitive composite scores.

\section{Individual variability analysis}

Within the high-intensity group only, changes in cardiorespiratory fitness were associated with changes in global cognitive function $\left(F=4.91, p<0.05, \eta_{\mathrm{p}}{ }^{2}=0.18\right)$ and executive function $\left(F=13.89, p<0.001, \eta_{\mathrm{p}}{ }^{2}=0.37\right.$; Table 3$)$. Increases in cardiorespiratory fitness were associated with improvements in global cognition $\left(F=4.37, p<0.05, \eta_{\mathrm{p}}{ }^{2}=\right.$ $0.06)$ and executive function $\left(F=4.83, p<0.05, \eta_{\mathrm{p}}{ }^{2}=0.06\right)$ from pre- to post-intervention in the whole sample.

The $B D N F^{*}$ fitness change interaction term was significant for global cognition $\left(F=5.13, p<0.05, \eta_{\mathrm{p}}{ }^{2}=0.07\right)$, executive function $\left(F=4.54, p<0.05, \eta_{\mathrm{p}}{ }^{2}=0.06\right)$, and episodic memory $\left(F=4.96, p<0.05, \eta_{\mathrm{p}}{ }^{2}=0.07\right)$. Post hoc analyses of these interactions revealed non-Met carriers (i.e. BDNF Val/Val homozygotes) received benefit in terms of a relationship between change in cardiorespiratory fitness and global cognitive function $(F=7.52, p<$ $\left.0.01, \eta_{\mathrm{p}}{ }^{2}=0.16\right)$ and executive function $(F=8.83, p<$ $0.01, \eta_{\mathrm{p}}{ }^{2}=0.18$; Fig. $2 \mathrm{a}-\mathrm{c}$ ), i.e. greater improvements in cardiorespiratory fitness were associated with greater improvements in cognitive performance post-intervention among non-Met carriers. 
Table 2 Effects of the exercise interventions on physiological measures and cognitive composite scores

\begin{tabular}{|c|c|c|c|c|c|}
\hline & \multicolumn{3}{|c|}{ Raw mean change from baseline $(95 \% \mathrm{Cl})$} & \multicolumn{2}{|c|}{$\begin{array}{l}\text { Time*Group (ITT) } \\
\text { Unstandardized B (standard error) }\end{array}$} \\
\hline & High-intensity $(n=33)$ & Moderate-intensity $(n=34)$ & Control $(n=32)$ & Baseline to 6 months & All timepoints \\
\hline \multicolumn{4}{|c|}{$\mathrm{VO}_{2}$ peak $(\mathrm{ml} / \mathrm{kg} / \mathrm{min})$} & $3.67(0.72)^{* *}$ & $0.35(0.38)$ \\
\hline 6 & $5.40(4.00,6.81)$ & $3.02(1.79,4.25)$ & $0.55(-0.67,1.77)$ & & \\
\hline 18 & $0.78(-0.62,2.18)$ & $-1.43(-2.78,-0.08)$ & $-0.99(-2.85,0.86)$ & & \\
\hline \multicolumn{4}{|c|}{ Peak power (Watts) } & $27.33(4.24)^{* *}$ & $3.18(2.51)$ \\
\hline 6 & $37.2(29.4,45.1)$ & $29.5(20.6,38.4)$ & $1.60(-3.12,6.33)$ & & \\
\hline 18 & $7.2(-0.6,15.1)$ & $0.14(-6.56,6.85)$ & $-9.59(-16.7,-2.43)$ & & \\
\hline \multicolumn{4}{|c|}{$\%$ Body fat } & $-0.80(0.37)^{*}$ & $-0.09(0.23)$ \\
\hline 6 & $-1.04(-1.83,-0.25)$ & $-0.48(-1.05,0.10)$ & $0.00(-0.64 .0 .63)$ & & \\
\hline 18 & $1.73(0.39,3.07)$ & $2.64(1.85,3.44)$ & $1.97(0.70,3.24)$ & & \\
\hline \multicolumn{4}{|c|}{ Global Cognitive composite } & $-0.04(0.07)$ & $-0.02(0.03)$ \\
\hline 6 & $0.11(-0.03,0.24)$ & $0.19(0.09,0.28)$ & $0.13(-0.02,0.27)$ & & \\
\hline 18 & $0.18(0.05,0.30)$ & $0.21(0.07,0.34)$ & $0.23(0.08,0.38)$ & & \\
\hline \multicolumn{4}{|c|}{ Executive function composite } & $0.00(0.09)$ & $0.02(0.04)$ \\
\hline 6 & $0.17(-0.00,0.34)$ & $0.31(0.14,0.49)$ & $0.13(-0.03,0.30)$ & & \\
\hline 18 & $0.30(0.12,0.47)$ & $0.31(0.06,0.56)$ & $0.18(0.00,0.35)$ & & \\
\hline \multicolumn{4}{|c|}{ Episodic memory composite } & $0.02(0.11)$ & $-0.04(-0.04)$ \\
\hline 6 & $0.19(-0.04,0.43)$ & $0.15(-0.00,0.30)$ & $0.13(-0.07,0.34)$ & & \\
\hline 18 & $0.20(-0.04,0.43)$ & $0.25(0.07,0.44)$ & $0.32(0.12,0.53)$ & & \\
\hline \multicolumn{4}{|c|}{ Attention composite } & $0.01(0.15)$ & $-0.03(0.05)$ \\
\hline 6 & $-0.17(-0.50,0.15)$ & $0.11(-0.12,0.35)$ & $-0.22(-0.48,0.04)$ & & \\
\hline 18 & $-0.09(-0.30,0.13)$ & $-0.02(-0.26,0.23)$ & $-0.10(-0.33,0.13)$ & & \\
\hline
\end{tabular}

We did not observe an effect of $A P O E^{*}$ fitness change on any of the cognitive change scores (Table 3). However, following stratification by $A P O E$ \&4 carriage, only $\varepsilon 4$ carriers demonstrated an association between increases in cardiorespiratory fitness and improvements in global cognition $\left(F=4.92, p<0.05, \eta_{\mathrm{p}}{ }^{2}=0.23\right.$; eFigure 2$)$.

\section{Discussion}

The current study compared the impact of 6 months of supervised high- and moderate-intensity exercise on cognition in a group of cognitively normal older adults. Our intervention successfully delivered high-intensity exercise and resulted in greater increases in cardiorespiratory

Table 3 Changes in cardiorespiratory fitness (residuals) and cognitive function (residuals) from pre- to immediately post-intervention

\begin{tabular}{|c|c|c|c|c|}
\hline \multirow{3}{*}{ Dependent variable $^{a}$} & \multicolumn{4}{|c|}{ Independent variable ( $F$ statistic) } \\
\hline & \multirow{2}{*}{$\begin{array}{l}\text { High-intensity } \\
\text { group } \\
\text { Fitness change }^{a}\end{array}$} & \multicolumn{3}{|c|}{ Whole cohort } \\
\hline & & $\begin{array}{l}\text { Fitness } \\
\text { change }^{a}\end{array}$ & $\begin{array}{l}\text { Fitness change }{ }^{\mathrm{a}} \text { x BDNF } \\
\text { Val66Met }\end{array}$ & $\begin{array}{l}\text { Fitness change }{ }^{\mathrm{a}} \times A P O E \varepsilon 4 \\
\text { carriage }\end{array}$ \\
\hline Global Cognition change & $4.91 *$ & $4.37^{*}$ & $5.13^{*}$ & 2.50 \\
\hline $\begin{array}{l}\text { Executive Function } \\
\text { change }\end{array}$ & $13.89 * *$ & $4.83^{*}$ & $4.54^{*}$ & 0.33 \\
\hline Episodic Memory change & 0.68 & 0.84 & $4.96 *$ & 2.57 \\
\hline Attention change & 0.94 & 2.23 & 0.01 & 0.13 \\
\hline
\end{tabular}

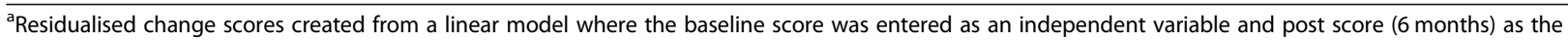
dependent variable. ${ }^{*} p<0.05,{ }^{* *} p<0.001$. Covariates: age, gender, years of education. Abbreviations: APOE apolipoprotein $\mathrm{E}, \mathrm{BDNF}$ Val66Met brain-derived neurotrophic factor Valine66Methionine single nucleotide polymorphism 

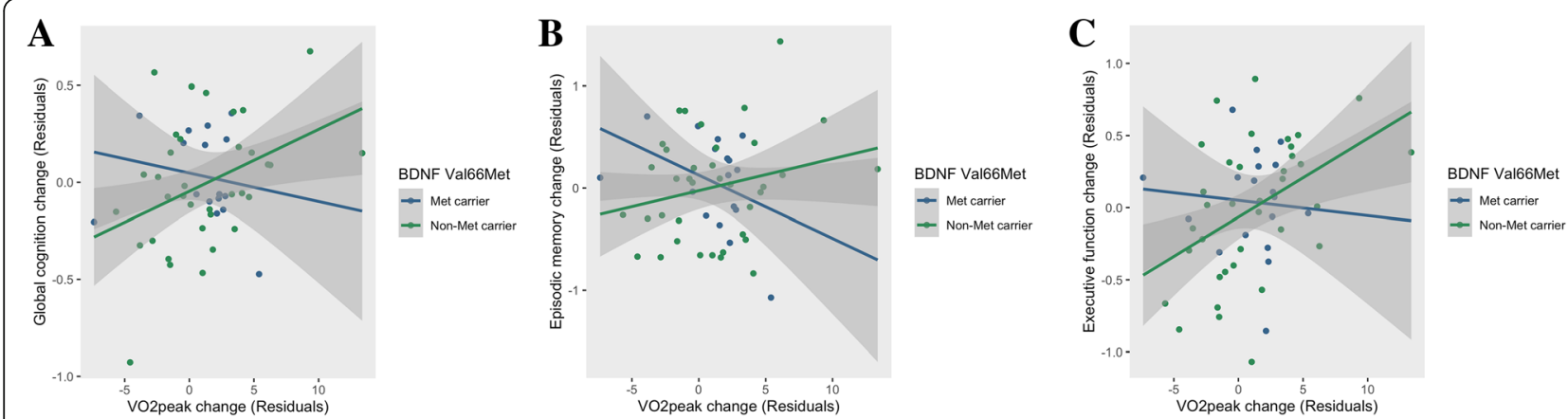

Fig. 2 Linear relationships between change in cardiorespiratory fitness (residuals) and change in a global cognition (residuals), b episodic memory (residuals), and c executive function (residuals); from pre- to immediately post-intervention (6 months) in BDNF Val66Met carriers and non-Met carriers. Abbreviations: BDNF Val66Met, brain-derived neurotrophic factor Valine66Methionine single nucleotide polymorphism; $\mathrm{VO}_{2}$ peak, peak aerobic capacity (fitness measurement)

fitness, compared with the moderate-intensity group. We did not observe any beneficial effects to cognition when comparing group performance from pre- to postintervention. However, changes in cardiorespiratory fitness from pre- to post-intervention were associated with changes in global cognition and executive function in the high-intensity group, and the entire cohort. From exploratory genetic analyses, we observed moderating effects of the $B D N F$ Val66Met polymorphism, whereby the relationship between change in cognition and change in fitness was only evident in Val/Val homozygotes (i.e. non-Met carriers).

When examining the group-level data, we found no effect of either the high- or moderate-intensity exercise interventions on cognitive performance. These findings are inconsistent with a recent RCT which demonstrated improvements on a single high-interference memory task, following a 12-week high-intensity exercise intervention, compared to moderate-intensity exercise [8]. As the cohort within the current study and that investigated by Kovacevic et al. were similar for age, cognitive status, and health, a methodological difference between studies may instead account for the disparate findings. It is possible that more frequent exercise, at least thrice-weekly (delivered by Kovacevic and colleagues), is required to induce cognitive benefit, even with a shorter intervention period (12 weeks). Indeed, the induction of neurotrophic factors (e.g. BDNF) may be required on a more 'frequent' basis to contribute to detectable neural benefits [21]. It would not be surprising that duration and frequency, in addition to intensity, play an important role in exercise-induced cognitive response. Thus, future studies are required to further elucidate the optimal exercise parameters for benefiting cognition.

Within the entire cohort, and high-intensity group alone, changes in cardiorespiratory fitness were associated with changes in global cognitive performance and executive function from pre- to post-intervention, with moderate to high effect sizes observed. These data support prior work that has reported relationships between exercise-induced improvements in cardiorespiratory fitness and cognitive changes $[13,20,22,23]$. The highintensity exercise intervention in the current study increased cardiorespiratory fitness levels greater than prior similar RCTs in older adults (typically $10-15 \%$ increases) $[8,13,24]$. It therefore remains puzzling as to why the observed associations between changes in cardiorespiratory fitness and cognition did not yield group-level differences in cognitive outcomes. While it is possible that individuals with the poorest baseline cognition and fitness levels were more likely to experience fitnessassociated cognitive improvement irrespective of exercise intervention, our statistical analysis at least partially corrected for this potential bias. It is also important to consider whether the cognitive assessments used within this generally high-functioning sample were sensitive enough to detect differences between groups.

We conducted an exploratory analysis to examine whether important genetic factors play a role in determining cognitive response following the delivered exercise intervention, and it is important to note that these analyses may have been underpowered. The associations between changes in cardiorespiratory fitness and global cognition, executive function, and episodic memory were strongest in $B D N F \mathrm{Val} / \mathrm{Val}$ homozygotes. Increases in BDNF levels are one of the most well-supported mechanistic theories underlying the relationship between exercise and brain health [25]. BDNF is synthesised in cells as a precursor molecule (pro-BDNF), which undergoes proteolytic cleavage to yield the mature form. Carriage of the BDNF Met allele can negatively alter the processing of pro-BDNF to mature BDNF in neurons: validation of our preliminary findings by future work would suggest that exercise may not be potent enough to counteract this detrimental phenotype. Consistent with our findings, a recent systematic review on this topic 
revealed $\mathrm{Val} / \mathrm{Val}$ homozygotes are more likely to gain benefit from exercise in terms of better memory performance, compared with Met carriers [26]. Moreover, we also observed a relationship between fitness and global cognition in $A P O E$ \&4 carriers, but not non-carriers; however, the interaction term $A P O E^{*}$ fitness change was not significant (indicating that a moderating effect does not exist). It is likely that combinations of genetic factors influence the relationship between cardiorespiratory fitness and cognition. Previous studies have detected cumulative effects of $A P O E \quad \varepsilon 4$ carriage and $B D N F$ Val66Met carriage on cognitive decline [27]; however, our study was not sufficiently powered to examine the $A P O E * B D N F$ interaction. Consequently, appropriately powered exercise interventions coupled with hypothesisdriven genetic investigation may reveal more on the ability of exercise to either provide added benefit to those with optimal genetic factors, or alternatively counteract detrimental genotypes.

\section{Strengths and limitations}

The lack of an active control group within the current study contributed to differences in exposure (e.g. social interactions) between our exercise groups and inactive control groups. Nevertheless, as we did not detect group differences across cognitive outcomes, this is unlikely to have affected the results reported here. Our cohort was a generally homogenous sample of highly educated, Caucasian older adults living in the community, and our results may not be applicable to the wider population. Strengths of the study include the three-group design that aimed to detect intensity differences, and our ability to objectively examine intensity levels throughout the intervention. Indeed, our use of rate of perceived exertion to monitor within-subject intensity proved to be an effective method in our older adult cohort, allowing individuals to maintain appropriate intensity targets without the need for frequent testing or the use of monitoring equipment (e.g. heart rate monitors).

\section{Conclusions}

We found that in a cohort of cognitively normal older adults, 6 months of supervised high-intensity and moderate-intensity exercise did not directly contribute to improvements in cognition, compared to an inactive control group. Our data did, however, demonstrate that changes in cardiorespiratory fitness were associated with cognitive change from pre- to post-intervention. Furthermore, our exploratory analysis of important genetic factors provided preliminary evidence that $A P O E$ and $B D N F$ genotypes may affect the relationship between cardiorespiratory fitness and cognitive change. Overall, our data does not provide evidence that high-intensity exercise can contribute to cognitive change in all individuals. Future work in this field should be appropriately designed and powered to examine numerous factors that could contribute to individual variability in response to intervention, ultimately leading to individualised prescription of exercise to induce cognitive change and ultimately reduce dementia risk.

\section{Supplementary Information}

The online version contains supplementary material available at https://doi. org/10.1186/s13195-021-00774-y.

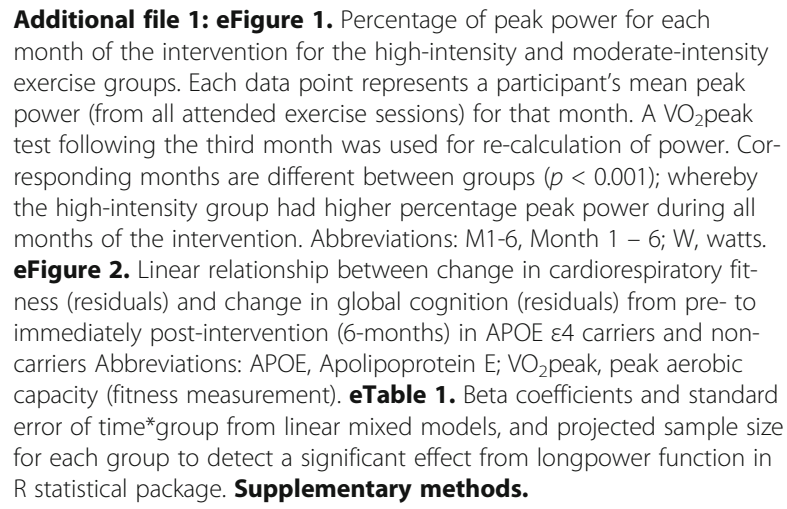

\section{Acknowledgements}

All authors would like to thank the IPAC study participants for their generous participation and contribution of time.

\section{Authors' contributions}

BB designed the study, collected the data, analysed the data, and drafted the manuscript; NF collected the cognitive data and provided a critical analysis of the manuscript; SRS was involved in the study design and critical review of the manuscript; JD assisted with the analytic design and provided biostatistical oversight and a critical review of the manuscript; SM collected the cognitive data and provided a critical review of the manuscript; NG delivered the exercise intervention, collected the physical test data, and provided a review of the manuscript; MW designed the cognitive test battery and provided critical review of the manuscript; HRS designed the cognitive battery, assisted with cognitive test interpretation, and provided a critical review of the manuscript; SML conducted genotyping and provided a critical review of the manuscript; RNM was involved in the study design and critical review of the manuscript; KIE was involved in the analytical design and provided a critical review of the manuscript; JP was involved in the study design, provided oversight of exercise delivery, interpretation of study findings, and provided a critical review of the manuscript. The authors read and approved the final manuscript.

\section{Funding}

The IPAC study is supported by a National Health and Medical Research Council Dementia Research Development Fellowship awarded to Belinda M Brown (grant number: GNT1097105)

\section{Availability of data and materials}

The datasets generated and/or analysed during the current study are not publicly available due to additional secondary analyses currently being conducted, but are available from the corresponding author on reasonable request.

\section{Ethics approval and consent to participate}

The IPAC study is registered with the Australian New Zealand Clinical Trials Registry (ACTRN12617000643370). The human research ethics committees at Murdoch University and Edith Cowan University approved the conduct of this study, and all participants provided written informed consent. 


\section{Consent for publication}

Not applicable.

\section{Competing interests}

The authors declare that they have no competing interests.

\section{Author details}

'Discipline of Exercise Science, College of Science, Health, Engineering and Education, Murdoch University, 90 South Street, Murdoch, WA 6150, Australia. ${ }^{2}$ Australian Alzheimer's Research Foundation, Sarich Neuroscience Research Institute, Nedlands, Western Australia, Australia. ${ }^{3}$ Centre for Healthy Ageing, Murdoch University, Murdoch, Western Australia, Australia. ${ }^{4}$ School of Medical and Health Sciences, Edith Cowan University, Joondalup, Western Australia, Australia. ${ }^{5}$ School of Psychological Science, University of Western Australia, Crawley, Western Australia, Australia. ${ }^{6}$ Australian eHealth Research Centre, CSIRO, Brisbane, Queensland, Australia. ${ }^{7}$ Department of Biomedical Sciences, Macquarie University, Sydney, New South Wales, Australia. ${ }^{8}$ Collaborative Genomics and Translation Group, School of Medical and Health Sciences, Edith Cowan University, Joondalup, Western Australia, Australia. ${ }^{9}$ School of Pharmacy and Biomedical Sciences, Faculty of Health Sciences, Curtin Health Innovation Research Institute, Curtin University, Bentley, Western Australia, Australia. ${ }^{10}$ Department of Psychology, University of Pittsburgh, Pittsburgh, PA, USA.

Received: 18 September 2020 Accepted: 15 January 2021 Published online: 01 February 2021

\section{References}

1. Norton S, Matthews FE, Barnes DE, Yaffe K, Brayne C. Potential for primary prevention of Alzheimer's disease: an analysis of population-based data. Lancet Neurol. 2014;13(8):788-94.

2. Young J, Angevaren M, Rusted J, Tabet N. Aerobic exercise to improve cognitive function in older people without known cognitive impairment. Cochrane Database Syst Rev. 2015:4:CD005381

3. Northey JM, Cherbuin N, Pumpa KL, Smee DJ, Rattray B. Exercise interventions for cognitive function in adults older than 50: a systematic review with meta-analysis. Br J Sports Med. 2018;52(3):154-60.

4. Brown BM, Peiffer JJ, Sohrabi HR, Mondal A, Gupta VB, Rainey-Smith SR, et al. Intense physical activity is associated with cognitive performance in the elderly. Transl Psychiatry. 2012;2:e191.

5. Angevaren $M$, Vanhees $L$, Wendel-Vos W, Verhaar HJ, Aufdemkampe G, Aleman A, et al. Intensity, but not duration, of physical activities is related to cognitive function. Eur J Cardiovasc Prev Rehabil. 2007;14(6):825-30.

6. Tsukamoto H, Suga T, Takenaka S, Tanaka D, Takeuchi T, Hamaoka T, et al. Greater impact of acute high-intensity interval exercise on post-exercise executive function compared to moderate-intensity continuous exercise. Physiol Behav. 2016;155:224-30.

7. Alves CR, Tessaro VH, Teixeira LA, Murakava K, Roschel H, Gualano B, et al. Influence of acute high-intensity aerobic interval exercise bout on selective attention and short-term memory tasks. Percept Mot Skills. 2014;118(1):63-72.

8. Kovacevic A, Fenesi B, Paolucci E, Heisz JJ. The effects of aerobic exercise intensity on memory in older adults. Appl Physiol Nutr Metab. 2019;45(6):110.

9. Keating CJ, Parraga Montilla JA, Latorre Roman PA, Moreno Del Castillo R. Comparison of high-intensity interval training to moderate-intensity continuous training in older adults: a systematic review. J Aging Phys Act. 2020;28(5):1-10.

10. Brown BM, Bourgeat P, Peiffer JJ, Burnham S, Laws SM, Rainey-Smith SR, et al. Influence of BDNF Val66Met on the relationship between physical activity and brain volume. Neurology. 2014;83(15):1345-52.

11. Brown BM, Castalanelli N, Rainey-Smith SR, Doecke J, Weinborn M, Sohrabi $H R$, et al. Influence of BDNF Val66Met on the relationship between cardiorespiratory fitness and memory in cognitively normal older adults. Behav Brain Res. 2019:362:103-8.

12. Brown BM, Peiffer JJ, Taddei K, Lui JK, Laws SM, Gupta VB, et al. Physical activity and amyloid-beta plasma and brain levels: results from the Australian Imaging, Biomarkers and Lifestyle Study of Ageing. Mol Psychiatry. 2013;18(8):875-81

13. Vidoni ED, Johnson DK, Morris JK, Van Sciver A, Greer CS, Billinger SA, et al. Dose-response of aerobic exercise on cognition: a community-based, pilot randomized controlled trial. PLoS One. 2015;10(7):e0131647.
14. Komulainen P, Lakka TA, Kivipelto M, Hassinen M, Helkala EL, Haapala I, et al Metabolic syndrome and cognitive function: a population-based follow-up study in elderly women. Dement Geriatr Cogn Disord. 2007;23(1):29-34.

15. Brown BM, Rainey-Smith SR, Castalanelli N, Gordon N, Markovic S, Sohrabi $H R$, et al. Study protocol of the Intense Physical Activity and Cognition study: the effect of high-intensity exercise training on cognitive function in older adults. Alzheimers Dement (New York, N Y). 2017;3(4):562-70.

16. Schulz KF, Altman DG, Moher D, Group C. CONSORT 2010 statement: updated guidelines for reporting parallel group randomised trials. Trials. 2010;11:32.

17. Borg G. Borg's perceived exertion and pain scales: human kinetics; 1998

18. Porter T, Villemagne $V L$, Savage G, Milicic L, Lim YY, Maruff $P$, et al. Cognitive gene risk profile for the prediction of cognitive decline in presymptomatic Alzheimer's disease. Personalized Med Psychiatry. 2018;7:14-20.

19. R Core Team. R: A language and environment for statistical computing. $R$ Foundation for Statistical Computing, Vienna, Austria. 2020. https://www.Rproject.org/.

20. Voss MW, Heo S, Prakash RS, Erickson Kl, Alves H, Chaddock L, et al. The influence of aerobic fitness on cerebral white matter integrity and cognitive function in older adults: results of a one-year exercise intervention. Hum Brain Mapp. 2013;34(11):2972-85.

21. Marston KJ, Newton MJ, Brown BM, Rainey-Smith SR, Bird S, Martins RN, et al. Intense resistance exercise increases peripheral brain-derived neurotrophic factor. J Sci Med Sport. 2017;20(10):899-903.

22. Reiter K, Nielson KA, Smith TJ, Weiss LR, Alfini AJ, Smith JC. Improved cardiorespiratory fitness is associated with increased cortical thickness in mild cognitive impairment. J Int Neuropsychol Soc. 2015;21(10):757-67.

23. Komulainen $P$, Kivipelto M, Lakka T, Savonen K, Hassinen M, Kiviniemi V, et al. Exercise, fitness and cognition-a randomised controlled trial in older individuals: the DR's EXTRA study. Eur Geriatr Med. 2010;1(5):266-72.

24. Fenesi B, Fang H, Kovacevic A, Oremus M, Raina P, Heisz JJ. Physical exercise moderates the relationship of apolipoprotein E (APOE) genotype and dementia risk: a population-based study. J Alzheimers Dis. 2017;56(1):297-303.

25. Liu PZ, Nusslock R. Exercise-mediated neurogenesis in the Hippocampus via BDNF. Front Neurosci. 2018;12:52

26. Liu T, Li H, Colton JP, Ge S, Li C. The BDNF Val66Met polymorphism, regular exercise, and cognition: a systematic review. West J Nurs Res. 2020;42(8): 193945920907308

27. Lim YY, Villemagne VL, Laws SM, Pietrzak RH, Snyder PJ, Ames D, et al. APOE and BDNF polymorphisms moderate amyloid beta-related cognitive decline in preclinical Alzheimer's disease. Mol Psychiatry. 2015;20(11):1322-8.

\section{Publisher's Note}

Springer Nature remains neutral with regard to jurisdictional claims in published maps and institutional affiliations.

Ready to submit your research? Choose BMC and benefit from:

- fast, convenient online submission

- thorough peer review by experienced researchers in your field

- rapid publication on acceptance

- support for research data, including large and complex data types

- gold Open Access which fosters wider collaboration and increased citations

- maximum visibility for your research: over $100 \mathrm{M}$ website views per year

At BMC, research is always in progress.

Learn more biomedcentral.com/submissions 